\title{
Uso de harina de Colocasia esculeta L., en la alimentación de cerdos y su efecto sobre parámetros productivos
}

\section{Using Colocasia esculenta L. meal in feed for pigs and its effect on production parameters}

\author{
Aragadvay-Yungán Ramón Gonzalo*, Núñez-Torres Oscar Patricio, Velástegui-Espín Giovanny Patricio, \\ Villacís-Aldaz Luis Alfredo, Guerrero-López Jorge Ricardo
}

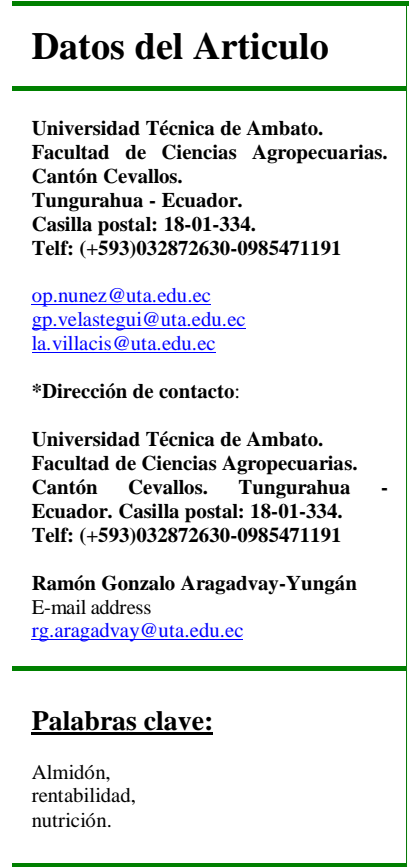

\section{J. Selva Andina Anim Sci.} 2016; 3(2):98-104.

\section{Historial del artículo. \\ Recibido febrero, 2016. \\ Devuelto agosto 2016 \\ Aceptado septiembre, 2016 \\ Disponible en línea, octubre, 2016

\begin{tabular}{c}
\hline Editado por: \\
Selva Andina \\
Research Society \\
\hline
\end{tabular}

\section{Key words:}

Starch,

Profitability,

nutrition.

\section{Resumen}

La presente investigación se la realizó con el objetivo de determinar los efectos sobre los parámetros productivos al incorporar harina de papa china (Colocasia esculenta L.) como materia prima en la elaboración de dietas para cerdos post destete, tomando en cuenta también los costos de producción. En este sentido se desarrolló tres formulaciones balanceadas con inclusión del 10, 20 y 30\% de harina de Colocasia esculenta L. (B1-B2 y B3 respectivamente), para ser comparadas frente a una formulación convencional (B0). Se utilizaron 16 cerdos raza Large White $\mathrm{x}$ Pietrain de 30 días de edad y un peso vivo entre 7-10 kg. El experimento se llevó a cabo bajo un diseño de parcelas divididas, distribuidos en 4 tratamientos y 4 repeticiones, con dos réplicas. Los resultados obtenidos se analizaron con el paquete estadístico MiniTab® 2000. En cuanto a los resultados, se reporta un peso final de $25.28 \mathrm{Kg}$, correspondiente al tratamiento B2 (20 \% de harina Colocasia esculenta L), mientras que para B1, B0 y B3 se encontró pesos de $24.19 \mathrm{~kg}$; 23,44 kg; y $22.87 \mathrm{~kg}$ respectivamente. Un comportamiento similar se observó en la ganancia de peso, mostrando B2: $15.93 \mathrm{~kg}$, diferente estadísticamente $(\mathrm{p}<0.05)$ frente a $14.47 \mathrm{~kg}, 13.88 \mathrm{~kg}$ y $13.36 \mathrm{~kg}$ correspondiente a B1, B0 y B3. En lo que refiere a la conversión alimenticia B2 mostró un valor de 1,30 que difirió estadísticamente ( $\mathrm{p}<0.05)$ al compararlas con B0: 1.39; B1: 1.43 y B3: 1.48. Por otra parte el tratamiento B3 presentó un beneficio neto superior a los demás tratamientos con valor de $\$ 338.17$.

(C) 2016. Journal of the Selva Andina Animal Science. Bolivia. Todos los derechos reservados.

\section{Abstract}

This investigation was made in order to determine the effects on production by incorporating Chinese potato flour olocasia esculenta L.) as a raw material in the production of pig diets post weaning parameters, taking into account duction costs. In this sense balanced three formulations including 10, 20 and 30\% flour Colocasia esculenta L. (B1-B2 $\mathrm{A} 33$ respectively), to be compared against a standard formulation (B0) was developed. 16 pigs Large White x Pietrain 30 ys old and live weight 7-10 kg were used. The experiment was conducted under a split plot design, distributed in 4 atments and 4 replicates with two replicas. The results were analyzed with the statistical package Minitab 2000. As for the ults, a final weight of $25.28 \mathrm{~kg}$ is reported, for the treatment B2 (20\% flour Colocasia esculenta L), while for B1, B0 B3 $\mathrm{I}$ weights of $24.19 \mathrm{~kg}$ was found; $23.44 \mathrm{~kg}$; and $22.87 \mathrm{~kg}$ respectively. A similar behavior was observed in weight gain, wwing B2: $15.93 \mathrm{~kg}$, statistically different ( $\mathrm{p}<0.05)$ versus $14.47 \mathrm{~kg}, 13.88 \mathrm{~kg}$ and $13.36 \mathrm{~kg}$ corresponding to B1, B0 and . As regards B2 feed conversion showed a value of 1.30 that differ statistically ( $\mathrm{p}<0.05$ ) when compared with B0: 1.39, : B3 1.43 and 1.48. Moreover, the treatment B3 showed a net profit than other treatments worth $\$ 338.17$. 


\section{Introducción}

La búsqueda de alimentos no convencionales para su uso como alternativas en la alimentación animal se ha incrementado en los últimos años (Gabbi et al. 2013, García-Martínez et al. 2009, Arriaga-Jordán et al. 2002) con la finalidad común de reducir los costos por alimentación. En este sentido el uso de tubérculos como Solanum tuberosum, Manihot esculenta, Ipomoea batata y Xanthosoma sagittifolium en la alimentación animal se vuelven una alternativa, por ser cultivos que representan a nivel mundial el segundo lugar en área sembrada en el planeta (Montaldo 1991).

Por otra parte la Colocasia esculeta L, también forma parte de la familia de tubérculos que por sus altos contenidos de almidón y fibra dietética (Sintagma et al. 2015) ha sido utilizada en la alimentación humana y con gran potencial como alimento animal especialmente en cerdos (Caicedo et al. 2014). En este tenor Tepper et al. (2012) menciona que la actualidad las actividades productivas que se dedican a la explotación porcina depende en gran porcentaje de la alimentación las mismas que al depender de insumos externos como alimento balanceado comercial, reducen el margen de ganancia mínima.

La Colocasia esculeta $\mathrm{L}$, es considerada una fuente barata de carbohidratos en relación a los cereales u otros cultivos de tubérculos (Aro 2012), además es altamente digerible ya que posee almidón con un diámetro menor (1-6.5 $\mu \mathrm{m}$ ) en comparación de otros tubérculos (Griffin \& Wang 1983), sin embargo este tubérculo al no recibir ningún tipo de tratamiento o cocción, en estado natural tienen un alto contenido de cristales de oxalato de calcio que son la causa de irritación, sensación de ardor en la boca y la garganta (Caicedo et al. 2015). El contenido de oxalato de 99 calcio varía con la especie y cultivares, forman complejos con las proteínas e inhiben su absorción y digestión, afectando de esta manera el normal crecimiento de animales (Caicedo et al. 2014).

En este sentido se observa que al recibir un tratamiento previo antes de ser suministrado a los animales se modifica su composición nutricional, elevando el contenido de materia seca y proteína cruda si es suministrado en forma de harina (Caicedo et al. 2014), convirtiéndose en un alimento alternativo que sustituya en gran porcentaje a los alimentos convencionales (Brunori 2009).

Bajo estas premisas el objetivo de esta investigación fue utilizar como alimento no convencional la harina de papa china (Colocasia esculeta L.) con diferentes niveles de inclusión (0-10-20 y 30\%) en dietas para la alimentación de cerdos destetados para determinar sus efectos sobre los parámetros productivos y de rentabilidad.

\section{Materiales y métodos}

El trabajo de campo de la presente investigación se realizó en la Parroquia Tarqui, Provincia Pastaza, Ecuador, en el Km 1.5 de la vía Tarqui-Madre Tierra. Sus coordenadas geográficas son: $01^{\circ} 32^{\prime} 00^{\prime \prime}$ de latitud Sur y $78^{\circ} 00^{\prime} 00^{\prime \prime}$ Oeste, está ubicado a una altura de $960 \mathrm{msnm}$ y una temperatura ambiental de $21{ }^{\circ} \mathrm{C}$. (IGM 2016). Se procedió al procesamiento de la harina de papa china a partir de la cosecha, de tubérculos cultivados en la zona de estudio posteriormente secado a estufa de aire forzado a $60{ }^{\circ} \mathrm{C}$ durante $72 \mathrm{~h}$, y reducción de tamaño de partícula mediante un molino tipo martillo marca Wiley® con una malla de1 mm. Posteriormente la harina 
obtenida se analizó su composición bromatológica en el Laboratorio de análisis de alimentos y productos procesados Tnte. Hugo Ortiz-Pastaza (Tabla 1).

Tabla 1 Análisis químico de la harina de papa china

\begin{tabular}{cccc}
\hline Análisis & Método & Unidad & $\begin{array}{c}\text { Harina de } \\
\text { papa china }\end{array}$ \\
\hline Grasa. & MO-LSAIA-01.02 & $\%$ & 0.8 \\
Proteína & PEE-LASA-FQ-1 I & $\%$ & 4.8 \\
& AOAC 991.20 & & \\
Fibra & MO-LSAIA-01.04 & $\%$ & 3.65 \\
EM & MO-LSAIA-01.10 & Mcal/kg & 2.59 \\
Ca & MO-LSAIA-01.12 & $\%$ & 0.01 \\
P & MO-LSAIA-01.13 & $\%$ & 0.02 \\
\hline
\end{tabular}

Para los corrales experimentales se utilizó madera para la elaboración de espacios con una dimensión de $0.80 \mathrm{~m}$ x $1.20 \mathrm{~m}$ en piso plástico con su respectivo comedero tipo tolva marca IPASA $®$ y bebederos automáticos tipo chupón, tomando en cuenta las recomendaciones para el bienestar animal. Se colocaron identificaciones en cada unidad experimental con el fin de realizar el suministro del alimento sin errores el mismo que fue proporcionado en forma de harina. Por otro lado el proceso de selección de 16 animales se realizó basados en el peso vivo con la finalidad de formar grupos homogéneos con un peso promedio de 7 a $10 \mathrm{Kg}$, tomando en cuenta que todos los animales estén en los 30 días de edad y cumplan con las características fenotípicas propias de la raza de cerdos Large White x Pietrain, también se realizó su desparasitación con Ivermectina previo al inicio del periodo experimental. Se alimentó a los cerdos en los comederos en dos raciones diarias previamente pesadas a las 8:00 y 15:00 respectivamente basados en su peso vivo según las recomendaciones de Campabadal (2009), se tomó en cuenta al alimento ofrecido y rechazado cada $24 \mathrm{~h}$. Las variables en estudio fueron peso inicial, peso final, ganancia de peso, conversión alimenticia y beneficio/costos por tratamiento.

El peso inicial fue registrado al comienzo del periodo experimental mediante una balanza electrónica 100 marca TOLEDO® con capacidad de $100 \mathrm{~kg}$ y una precisión de $0.1 \mathrm{~g}$, el peso final fue tomado una vez transcurrido los 30 días de suministrar las dietas experimentales y registrada en $\mathrm{kg}$ para su posterior tabulación.

Para ganancia de peso se obtuvo de la diferencia entre el peso final y peso inicial correspondiente a la cantidad en $\mathrm{kg}$ que incrementaron los cerdos en la fase de investigación. Para la conversión alimenticia se realizó en base a la cantidad de $\mathrm{kg}$ de alimento consumidos por cada cerdo, para la ganancia de peso de cada animal durante todo el periodo de investigación. Finalmente para el análisis económico de cada uno de los tratamientos se utilizó el método de Perrín et al. 1988. Para los resultados de las variable productivas se utilizó el paquete estadístico MiniTab® 2000, y para la comparación de medias la prueba de Tukey con una probabilidad del 5\%

El experimento se llevó a cabo bajo un diseño de parcelas divididas, distribuidos en 4 tratamientos con 4 repeticiones en 2 épocas, con tres niveles de harina de papa china 10\%, 20\%, 30\% incluidas en una concentración balanceada elaborada en el software Lindo 6.1 para Windows (Tabla 2) basados en los requerimientos para cerdos que indican las Tablas Brasileñas.

\section{Resultados}

El peso inicial, los datos logrados indican diferencias no significativas $(p>0.05)$ en las dos réplicas que se efectuaron para este experimento, resultado similares a la edad de 30 días ya que fueron escogidos con ésta característica intencionalmente para el inicio del experimento. El coeficiente de variación para el peso inicial fue de $5.26 \%$ y la media de $9.553 \mathrm{Kg}$., como se detalla en la Tabla 3. 
Tabla 2. Balanceado con papa china en su formulación comercial

\begin{tabular}{lcccc}
\hline Materias Primas & B0 0\% Papa China & B1 20\% Papa China & B2 20\% Papa China & B3 20\% Papa China \\
\hline Maíz & 50 & 45 & 32 & 22.65 \\
Harina de papa china & 0 & 10 & 20 & 30 \\
Aceite palma & 4.65 & 4.65 & 4.65 & 4 \\
Torta de Soya 47\% & 29 & 30 & 31 & 32 \\
HCl lisina & 0.50 & 0.50 & 0.50 & 0.50 \\
D-L metionina 99\% & 0.15 & 0.15 & 0.15 & 0.15 \\
L-Treonina 98\% & 0.20 & 0.20 & 0.20 & 0.20 \\
Lincomicina 1,1\% & 0.05 & 0.05 & 0.05 & 0.05 \\
Antimicótico (Zetox) & 0.05 & 0.05 & 0.05 & 0.05 \\
Albumino silicato & 0.05 & 0.05 & 0.05 & 0.05 \\
Antioxidante & 0.05 & 0.05 & 1.50 & 1.50 \\
Leche en polvo & 1.50 & 1.50 & 6.39 & 5.39 \\
Afrecho de trigo & 11.39 & 5.39 & 1.40 & 1.30 \\
Fosfato mono cálcico & 0.02 & 0.30 & 1.20 & 1.30 \\
Carbonato de Calcio & 1.58 & 1.30 & 0.10 & 0.50 \\
Cloruro de colina 60\% & 0.10 & 0.10 & 0.21 & 0.50 \\
Premix cerdo & 0.50 & 0.50 & $\mathbf{1 0 0}$ & 0.21 \\
Sal yodada & 0.21 & 0.21 & & $\mathbf{1 0 0}$ \\
\hline TOTAL & $\mathbf{1 0 0}$ & & & \\
\hline
\end{tabular}

Tabla 3 Comparación de medias de parámetros productivos en cerdos alimentados con dietas balanceadas más la inclusión de tres niveles de Colocasia esculeta $\mathrm{L}$

\begin{tabular}{lcccccc}
\hline & \multicolumn{2}{c}{ Niveles de inclusión (\%) de Colocasia esculeta L } & \multicolumn{2}{c}{ CV. (\%) } \\
\hline Variable & $\mathbf{0}$ & $\mathbf{1 0}$ & $\mathbf{2 0}$ & $\mathbf{3 0}$ & \\
Peso inicial $(\mathrm{kg})$ & $9.55 \mathrm{a}$ & $9.12 \mathrm{a}$ & $9.25 \mathrm{a}$ & $9.33 \mathrm{a}$ & 5.26 \\
Peso final $(\mathrm{kg})$ & $23.44 \mathrm{bc}$ & $24.19 \mathrm{ab}$ & $25.28 \mathrm{a}$ & $22.87 \mathrm{c}$ & 3.78 \\
Ganancia de peso $(\mathrm{kg})$ & $13.88 \mathrm{bc}$ & $14.47 \mathrm{~b}$ & $15.93 \mathrm{a}$ & $13.36 \mathrm{c}$ & 5.21 \\
Conversión alimenticia & $1.39 \mathrm{ab}$ & $1.43 \mathrm{a}$ & $1.30 \mathrm{~b}$ & $1.48 \mathrm{a}$ & 5.44 \\
\hline
\end{tabular}

Por otro lado los datos del peso final, indican la existencia de diferencias altamente significativas $(\mathrm{p}<0.05)$ para los tratamientos, registrando B2 $(20$ $\%$ de harina de papa china) un valor de $25.28 \mathrm{Kg}$, seguido de B1, B0 y B3 que presentaron pesos finales de $24.19 \mathrm{~kg}, 23.44 \mathrm{~kg}$, y $22.87 \mathrm{~kg}$ respectivamente.

La ganancia de peso, el análisis de varianza reflejó diferencias altamente significativas $(p<0.05)$ entre tratamientos, B2 presentó un valor promedio de $15.93 \mathrm{Kg}$, mientras que en último lugar lo ocupa el balanceado B3 con $13.36 \mathrm{Kg}$.

Por otro lado, el consumo de alimento durante el periodo experimental fue similares en todos los tratamientos, en relacion a la cantidad ofrecida y el alimento rechazado. El consumo fue $20.171 \mathrm{Kg}$., en el análisis de varianza para la conversión alimenticia mostró diferencias estadísticas significativas entre tratamientos $(\mathrm{p}<0.05)$, presentando el tratamiento B3 un promedio de 1.484 , mientras que el último lugar lo ocupa B2 con un promedio de 1.309. En relación al análisis económico, los costos de producción varían en los ensayos Tabla 4 , se observa que el balanceado representa mayor gasto B1 (10 $\%$ de harina de papa china) con un valor de 468.09 dólares. 
Tabla 4 Cálculo de costos por tratamiento experimental

\begin{tabular}{lllccc}
\hline \multicolumn{2}{l}{ Tratamientos } & Costo lechón & Mano de obra & Alimento & Costos \\
\hline 1 & B0 & 400 & 20 & 46,02 & 466,02 \\
2 & B1 & 400 & 20 & 48,09 & 468,09 \\
3 & B2 & 400 & 20 & 46,47 & 466,47 \\
4 & B3 & 400 & 20 & 41,83 & 461,83 \\
\hline
\end{tabular}

Se determinó el presupuesto parcial, Tabla 5, utilizando la propuesta de Perrín et al. 1988, que considera el ingreso y los costos que varían en el ensayo, obteniendo como resultado el balanceado B3 (30\% de harina de papa china) alcanzó el mayor beneficio con un valor de \$ 338.17 y B1 fue el menor beneficio neto con un valor de \$331.91.

Tabla 5 Cálculo de presupuesto parcial por tratamiento experimental

\begin{tabular}{lllll}
\hline & & $\begin{array}{c}\text { Beneficio } \\
\text { de campo }\end{array}$ & $\begin{array}{c}\text { Total costo } \\
\text { que varían }\end{array}$ & $\begin{array}{c}\text { Beneficios } \\
\text { netos }\end{array}$ \\
\hline 1 & B0 & 800 & 466.02 & 333.98 \\
2 & B1 & 800 & 468.09 & 331.98 \\
3 & B2 & 800 & 466.47 & 333.53 \\
4 & B3 & 800 & 461.83 & 338.17 \\
\hline
\end{tabular}

\section{Discusión}

La adición del $20 \%$ y $30 \%$ de harina de Colocasia esculenta L., en dietas para la alimentación de cerdos ocasionó pesos finales superiores a niveles de inclusión del 10\%, debido quizá a los altos contenidos de almidón de éste tubérculo que lo convierte en un alimento digestible a nivel intestinal, consecuentemente las carbohidratos energéticos altamente disponibles como la glucosa permiten a los animales cubrir sus demandas energéticas, que se reflejan en ganancia de peso. Caicedo, et al. (2014), menciona en estudios de microscopia y luz láser en algunas variedades de papa china presentan almidones de 1$6.5 \mu \mathrm{m}$ de diámetro, comparado con el arroz, de aproximadamente $5 \mu \mathrm{m}$ siendo es el más fino de los almidones, esto convierte a este tubérculo en un alimento funcional, que puede ser incorporado en dietas de cerdos, incluso en etapas iníciales para lechones, ya que las condiciones del tracto gastrointestinal muestra mayor demanda en la absorción de nutrientes.

De igual forma la inclusión del 20\% de harina de Colocasia esculenta L., arrojó resultados superiores en la variable ganancia total de peso $(15.93 \mathrm{~kg})$ comportamiento relacionado posiblemente con el alto índice de solubilidad del almidón, que está en función al contenido de amilopectina y un menor tamaño del gránulo, facilitan el ingreso de agua a los espacios intermoleculares aumentando su solubilidad (Hwang \& Kokini 1992). Por otro lado el alto contenido de amilosa es importante en el almidón asociado a un mayor contenido de zonas semi cristalinas, produce una mejor estabilidad interna del gránulo del almidón (Imberty 1988), y consecuencia una alta disponibilidad en este tipo de tubérculos.

$\mathrm{Al}$ referirnos a la conversión alimenticia se observó un índice de 1.30 para B2 (20\% de harina de papa china) y que refleja el alto valor nutritivo de sus componentes como el almidón, polisacárido de reserva constituido por proporciones variables de amilosa y amilopectina que en función de esta proporción será la funcionalidad o propiedades del almidón (Fernández-Sevilla 2005). Además su contenido de almidón se ve reflejado en el alto valor 
energético del tubérculo (2.59 Mcal $\left./ \mathrm{kg}^{-1}\right)$ Tabla 1, también se observa que su bajo valor de fibra bruta la hace asimilable al estar relacionada con polisacáridos constituyentes de la pared celular como celulosa y hemicelulosa (Aragadvay-Yungán et al. 2014).Finalmente el contenido de proteína cruda de la harina de Colocasia esculenta L se eleva con relación al tubérculo sin procesar, valores semejantes a lo reportado por Caicedo et al. (2014), podría estar relacionado a una mayor disponibilidad de aminoácidos escenciales requeridos para el desarrollo muscular.

Los costos de alimentación del cerdo pueden representar entre el $70 \%$ y el $80 \%$ de los totales de producción, y la energía como nutriente o requerimiento, es el rubro de mayor impacto en la alimentación por lo que desde el punto de vista económico el tratamiento B3 (30 \% de harina de papa china) es el que presenta mayor beneficio neto con valor de $\$$ 338.17 teniendo menores costos.

En conclusión, niveles entre el 20 y $30 \%$ de inclusión de harina de papa china (Colocasia esculenta L) en dietas para alimentación de cerdos resulta en un incremento de las principales parámetros productivos, así como en la reducción de costos relacionados a este rubro, pudiéndose considerar un alimento alternativo en la alimentación porcina.

\section{Conflictos de intereses}

La investigación realizada no presenta conflictos de interés. Además el presente estudio ha cumplido las normas éticas para el manejo de animales en trabajos de investigación.

\section{Agradecimientos}

Los autores agradecen a la Universidad Técnica de Ambato Facultad de Ciencias Agropecuarias, por el apoyo técnico, científico y logístico realizado a la presente investigación.

\section{Literatura citada}

Aragadvay RG, Rayas-Amor AA, Heredia D, Estrada J, Martínez FE, Arriaga CM. In vitro evaluation of sunflower (Helianthus annuus L.) silage alone or combined with maize silage. Rev Mex Cienc Pecu 2015; 6(3):315-327.

Arriaga CM, Albarrán B, Espinoza A, García A, Castelán OA. On-farm comparison of feeding strategies based on forages for small-scale dairy production systems in the highlands of central México. Expl Agric. 2002; 38(1):375388.

Aro SO, Akinjokun OM. Características de la carne y la canal de cerdos en crecimiento alimentados con peladuras de yuca tratadas microbialmente. Arch Zootec. . 2012; 61(235):407-414.

Brunori J. Estrategia de manejos en sistemas intensivos de producción de cerdos a campo. Ediporc. 2009; 125(1):34-40.

Caicedo QW, Rodríguez BR, Valle RS. Una Reseña Sobre El Uso de Tubérculos de Papa China Colocasia Esculenta Conservados En Forma de Ensilaje Para Alimentar Cerdos." Rev Electron Vet. 2014; 15(1): 1-10.

Caicedo QW, Rodríguez BR, Valle RS. Composición Química Y Digestibilidad in Vitro de Ensilados de Tubérculos de Papa China (Colocasia Esculenta L) Destinados a La Alimentación de Cerdos. Rev Cuba Cienc Agrícola. 2015; 40(1):59-64. 
Campabadal C. Guía técnica para alimentación de cerdos. Costa Rica: Asociación Americana de Soya-IM. 2009; p. 46.

Fernández-Sevilla JM. Estructura y función de los hidratos de carbono: azúcares, almidón, glucógeno, celulosa. Hidratos de carbono y su aprovechamiento. 2005; 1-38.

Gabbi AM, McManus CM, Silva AV, Marques LT, Zanela MB, Stumpf MP, et al. Typology and physical-chemical characterization of bovine milk produced with different productions strategies. Agricultural Systems. 2013; 121:130-4.

García-Martínez A, Albarrán-Portillo B, CastelánOrtega O, Espinoza-Ortega A, Arriaga-Jordán C. Urea treated maize straw for small-scale dairy systems in the highlands of Central Mexico. Trop Anim Health Prod. 2009; 41(1): 1487-94.

Griffin G, Wang J. Industrial Uses of Taro: a review of Colocacia esculenta and its potentials. University of Hawai Press. Honolulu-Hawai. 1983; 301-312.

Hwang J, Kokini J. Contribution of the side branches to rheological properties of pectins. Carbohydrate polymers. 1992; 19(1):41-50.

IGM.gob.ec. Geo visualizador [sede Web]. Instituto Geográfico Militar; acceso 16 de septiembre de 2016]. Disponible en http:// http://www.geopor taligm.gob.ec/portal/index.php/visualizador/
Imberty A, Chanzy H, Pérez S, Buleon A, Tran V. The double-helical nature of the crystalline part of A-starch. J Mol Biol. 1988; 201(2): 365-378.

Minitab, Statistical Software (Version 13.32). Minitab Inc., Philadelphia, PA, USA. 2000.

Montaldo A. Cultivos de Raíces y Tubérculos Tropicales. Editorial IICA. Costa Rica. 1991; 1367.

Perrin R, Wilkelmann D, Moscardi E, Amaerhon J. La formulación de recomendaciones a partir de datos agronómicos. Ed. Revisada. México. 1988; p. 73.

Sintagma W, Rodríguez R, Lezcano P, Vargas J, Valle S. Efecto de la inocuidad del ensilado biológico de la papa china (Colocasia esculenta (L.) Schott) para la alimentación de cerdos. Rev Amaz Cienc Tecnol. 2015; 2(3):162-171.

Tepper R, González C, Figueroa R, Araque H, Sulbarán L. Efecto de la alimentación con recursos alternativos sobre la cría de cerdos en cama profunda. Av en Investig Agropecu. 2012; 16(2): 23-33. 